\title{
Generation of random waves in time-dependent extended mild-slope equations using a source function method
}

\author{
Gunwoo Kimª ${ }^{\mathrm{a}}$ Changhoon Lee ${ }^{\mathrm{b} *}$, Kyung-Duck $\operatorname{Suh}^{\mathrm{c}}$
}

a School of Civil, Urban, and Geosystem Engineering, Seoul National University, San 56-1, Shinlim-dong, Gwanak-gu, Seoul 151-742, South Korea

${ }^{\mathrm{b}}$ Department of Civil and Environmental Engineering, Sejong University, 98 Kunja-dong, Kwangjin-gu, Seoul 143-747, South Korea

c School of Civil, Urban, and Geosystem Engineering \& Engineering Research Institute, Seoul National University, San 56-1, Shinlim-dong, Gwanak-gu, Seoul 151-742, South Korea

* Corresponding author. Fax: +82-2-3408-3294.

E-mail addresses: maossi1@snu.ac.kr (G. Kim), clee@sejong.ac.kr (C. Lee), kdsuh@snu.ac.kr (K.-D. Suh). 


\begin{abstract}
We develop techniques of numerical wave generation in the time-dependent extended mild-slope equations of Suh et al. (1997) and Lee et al. (2003) for random waves using a source function method. Numerical results for both regular and irregular waves in one and two horizontal dimensions show that the wave heights and the frequency spectra are properly reproduced. The waves that pass through the wave generation region do not cause any numerical disturbances, showing usefulness of the source function method in avoiding re-reflection problems at the offshore boundary.
\end{abstract}

Keywords: Source function method; Extended mild-slope equation; Numerical generation of wave; Random waves; Numerical experiment

\title{
1. Introduction
}

The mild-slope equation, which was derived first by Berkhoff (1972), is able to predict the transformation of waves due to refraction, diffraction, shoaling, and reflection from deep to shallow water. Nishimura et al. (1983) and Copeland (1985) developed hyperbolic equations for regular waves. Smith and Sprinks (1975), Radder and 
Dingemans (1985), and Kubo et al. (1992) developed time-dependent mild-slope equations that are able to predict the transformation of random waves.

Recently, efforts have been made to improve the mild-slope equation by including higher-order bottom effect terms proportional to the bottom curvature and to the square of bottom slope, which were neglected in the derivation of the mild-slope equation. The resulting equations are called the extended mild-slope equations or modified mild-slope equations. These equations may be regarded as a complete form of the linear refractiondiffraction equation because it is derived by integrating the Laplace equation from bottom to mean water surface without neglecting any terms. In an elliptic form, Massel (1993) and Chamberlain and Porter (1995) extended the model of Berkhoff. In a hyperbolic form, Suh et al. (1997) extended the two models of Smith and Sprinks, and Radder and Dingemans, Lee et al. (1998) extended the model of Copeland, and Lee et al. (2003) extended the model of Kubo et al.

To predict the wave field in a concerned region using time-dependent wave equations, waves should be generated at the offshore boundary and propagate into the model domain. Waves reflected from the model domain should pass through the offshore boundary without any numerical distortions. Otherwise, the waves re-reflected at the 
offshore boundary may influence the numerical solution in the model domain. In order to avoid the re-reflection problem at the wave generation boundary, internal wave generation techniques have been widely used. Larsen and Dancy (1983) and Madsen and Larsen (1987) employed a line source method to generate waves for the Peregrine's (1967) Boussinesq equations and Copeland's mild-slope equations, respectively, by using a mass transport approach. Lee and Suh (1998) and Lee et al. (2001) corrected the line source method by using the energy transport approach for the Radder and Dingemans' mild-slope equations and the Nwogu's (1993) Boussinesq equations, respectively. In the mass and energy transport approaches, the phase and energy velocities are used as the velocities of water mass and wave envelope, respectively, at the wave generation line.

Recently, Wei et al. (1999) developed a source function method for generating waves in the Boussinesq equations of Peregrine and Nwogu. The method employs a source term added to the governing equations, either in the form of a mass source in the continuity equation or an applied pressure forcing in the momentum equations. They used the Green function method to solve the governing equation including a spatially distributed source term.

The line source method generates waves at a single point along the wave propagation 
direction, while the source function method generates waves at a source band consisting of several grids along the wave propagation direction. In the line source method, waves are assumed to be generated at either side of the source point and to propagate across the source point at the speed of energy velocity. In the source function method, waves are assumed to be generated at the source band and to propagate continuously. Therefore, both methods can generate waves successfully without interfering the waves crossing the wave generation line or band. The line source method yielded a solution with slight numerical noises in some wave equations such as Radder and Dingemans' mild-slope equations (Lee and Suh, 1998) and Nwogu's Boussinesq equations (Lee et al., 2001), while such noises were not found in the Copeland's mild-slope equations (Lee and Suh, 1998). However, the source function method yielded a smooth solution without any numerical noise in the Boussinesq equations of Nwogu (Wei et al., 1999).

In this study, to generate waves without any noise in the model domain, source functions are developed for the time-dependent extended mild-slope equations of Suh et al. (1997) and Lee et al. (2003). And, numerical experiments were conducted to verify the source function method. In section 2, a source function is derived for each model. In section 3, numerical experiments are conducted for generating desired incident waves, including monochromatic and random waves in one and two horizontal dimensions. In 
section 4, summaries and discussions are presented.

\section{Derivation of source function}

The time-dependent extended mild-slope equations of Suh et al. (1997) and Lee et al. (2003) are reduced to the Helmholtz equation in a constant-depth region. In this section, we first derive a source function for the Helmholtz equation which is then used to obtain source functions for Suh et al. and Lee et al.'s equations.

\subsection{Helmholtz equation with source function}

The Helmholtz equation is the governing equation for linear waves propagating on a flat bottom. The Helmholtz equation with a source function is given by:

$\nabla^{2} F+k^{2} F=f$

where $F$ is the wave property such as surface elevation or velocity potential, $k$ is the wave number, $f$ is the source function, and $\nabla$ is the horizontal gradient operator. For waves propagating obliquely with respect to the $x$-axis, we may have the following 
expressions for $F$ and $f$ :

$F(x, y)=\tilde{F}(x) \exp \left[i\left(k_{y} y+\varepsilon\right)\right]$

$f(x, y)=\tilde{f}(x) \exp \left[i\left(k_{y} y+\varepsilon\right)\right]$

where $k_{y}$ is the wave number in the $y$-direction and $\varepsilon$ is the wave phase. Substituting

Eqs. (2) and (3) into Eq. (1) yields an ordinary differential equation for $\tilde{F}$ with respect to $x$ :

$\frac{\partial^{2} \tilde{F}}{\partial x^{2}}+k_{x}^{2} \tilde{F}=\tilde{f}$

where $k_{x}$ is the wave number in the $x$-direction. We can find a non-homogeneous solution for Eq. (4) by following the method of Wei et al. (1999). A detailed procedure is given in the Appendix A. The source function can adopt a smooth Gaussian shape:

$\tilde{f}(x)=D \exp \left[-\beta\left(x-x_{s}\right)^{2}\right]$

where $D$ and $\beta$ are parameters associated with the magnitude and width, respectively, of the source function and $x_{s}$ is the location of the center of the source region. 
Introducing the Green's function method to the differential equation gives the wave property corresponding to the source function:

$\tilde{F}(x)=A D I \exp \left[i k_{x}\left|x-x_{s}\right|\right]$

where $A$ and $I$ are given by

$$
\begin{aligned}
& A=-\frac{i}{2 k_{x}} \\
& I=\sqrt{\frac{\pi}{\beta}} \exp \left(-\frac{k_{x}^{2}}{4 \beta}\right)
\end{aligned}
$$

The parameter $D$ can be determined by the target wave property and the parameter $\beta$. Although a large value of $\beta$ is preferred since the source region can become narrower, too narrow a source region may cause a poor representation of the source function in the discretized equation. We follow Wei et al.'s recommendation to take the width of the source function as half of the wavelength. If we neglect the source function where the value of the Gaussian function $\left(\exp \left[-\beta\left(x-x_{s}\right)^{2}\right]\right.$ ) is smaller than $\exp (-5)=0.0067$, the width parameter $\beta$ is determined as $\beta=80 /(\delta \bar{L})^{2}$, where the value of $\delta$ is in the range of 0.3 to 0.5 and $\bar{L}$ is the wavelength corresponding to the carrier frequency $\bar{\omega}$. 


\subsection{Source function for Suh et al.'s model}

The time dependent mild-slope equations of Suh et al. (1997) are given by

$$
\begin{aligned}
& \frac{\partial \eta}{\partial t}+\nabla \cdot\left(\frac{\bar{C} \bar{C}_{g}}{g} \nabla \phi\right)-\frac{\bar{\omega}^{2}-\bar{k}^{2} \bar{C} \bar{C}_{g}}{g} \phi-\frac{\bar{\omega}^{2}}{g}\left\{\bar{R}_{1}(\nabla h)^{2}+\bar{R}_{2} \nabla^{2} h\right\} \phi=0 \\
& \frac{\partial \phi}{\partial t}+g \eta=0
\end{aligned}
$$

where $\eta$ is the surface elevation, $\phi$ is the velocity potential at mean water level, and $\bar{C}$ and $\bar{C}_{g}$ are the phase speed and the group velocity, respectively, $g$ is the gravitational acceleration, $h$ is the water depth, and the over bar indicates the variables associated with the carrier angular frequency $\bar{\omega}$. If $\bar{R}_{1}=\bar{R}_{2}=0$, the above equations are reduced to the model of Radder and Dingemans (1985).

We consider waves on a flat bottom. Adding the source function $f_{S}\left(=D_{S} \exp \left[-\beta\left(x-x_{s}\right)^{2}\right\rfloor\right.$ to the right-hand side of Eq. (9), where the subscript $S$ implies the model of Suh et al., and eliminating $\eta$ in favor of $\phi$ give the following equation: 
$\frac{\partial^{2} \phi}{\partial t^{2}}-\bar{C} \bar{C}_{g} \nabla^{2} \phi+\left(\bar{\omega}^{2}-\bar{k}^{2} \bar{C} \bar{C}_{g}\right) \phi=-g f_{S}$

For a component wave whose angular frequency is $\omega$, the $y$-directional and temporal harmonic terms of $\phi(x, y, t)$ and $f_{S}(x, y, t)$ can be separated as

$\phi=\tilde{\phi} \exp \left[i\left(k_{y} y-\omega t\right)\right]$

$f_{S}=\tilde{f}_{S} \exp \left[i\left(k_{y} y-\omega t\right)\right]$

Substituting Eq. (12) and (13) into Eq. (11) yields the one-dimensional Helmholtz equation in terms of $\tilde{\phi}$ :

$\frac{\partial^{2} \tilde{\phi}}{\partial x^{2}}+k_{x}^{2} \tilde{\phi}=\frac{g}{\bar{C} \bar{C}_{g}} \tilde{f}_{S}$

where the right-hand-side term can be regarded as the source function $\tilde{f}$ in the Helmholtz Eq. (4), and the wave number $k$ is the wave number corresponding to the angular frequency $\omega$ in this model, which can be obtained by the dispersion relation of the model (Lee et al., 2004) as 


$$
\frac{k}{\bar{k}}=\sqrt{1+\frac{\bar{C}}{\bar{C}_{g}}\left[\left(\frac{\omega}{\bar{\omega}}\right)^{2}-1\right]}
$$

Following the procedures from Eq. (4) to (8), the velocity potential function $\tilde{\phi}(x)$ corresponding to the source function can be obtained as

$$
\tilde{\phi}(x)=\frac{g A D_{S} I}{\bar{C} \bar{C}_{g}} \exp \left[i k_{x}\left|x-x_{s}\right|\right]
$$

where $A$ and $I$ are given in Eqs. (7) and (8). On the other hand, the target velocity potential function $\tilde{\phi}(x)$ is given as

$$
\tilde{\phi}(x)=-i \frac{a g}{\omega} \exp \left[i k_{x}\left|x-x_{s}\right|\right]
$$

where $a$ is the wave amplitude. Thus, the parameter $D_{S}$ in Eq. (16) can be obtained by equating $\tilde{\phi}(x)$ in Eqs. (16) and (17) as

$$
D_{S}=-i \frac{a \bar{C} \bar{C}_{g}}{\omega A I} .
$$

Thus, the source function $f_{S}$ for Suh et al.'s model is obtained as 


$$
f_{S}=-i \frac{a \bar{C} \bar{C}_{g}}{\omega A I} \exp \left[-\beta\left(x-x_{s}\right)^{2}\right] \exp \left[i\left(k_{y} y-\omega t\right)\right]
$$

\subsection{Source function for Lee et al.'s model}

The equation of Lee et al. (2003) are given by

$$
\begin{aligned}
& \nabla \cdot\left(\bar{C} \bar{C}_{g} \nabla \xi\right)+\left\{\bar{k}^{2} \bar{C} \bar{C}_{g}+g \bar{u}_{1} \nabla^{2} h+g \bar{u}_{2}(\nabla h)^{2}\right\} \xi+i \nabla \cdot\left\{\frac{\overline{\partial\left(C C_{g}\right)}}{\partial \omega} \nabla \frac{\partial \xi}{\partial t}\right\} \\
& +i\left\{\frac{\partial\left(k^{2} C C_{g}\right)}{\partial \omega}+g \frac{\overline{\partial u_{1}}}{\partial \omega} \nabla^{2} h+g \frac{\overline{\partial u_{2}}}{\partial \omega}(\nabla h)^{2}\right\} \frac{\partial \xi}{\partial t}=0
\end{aligned}
$$

where $\xi$ is related to the water surface elevation $\eta$ by $\eta=\xi \exp (-i \bar{\omega} t)$. If $\bar{u}_{1}=\bar{u}_{2}=0$, the above equation is reduced to the model of Kubo et al. (1992).

We consider waves on a flat bottom. Adding the source function $f_{L}\left(=D_{L} \exp \left[-\beta\left(x-x_{s}\right)^{2}\right]\right.$ to the right-hand side of Eq. (20), where the subscript $L$ implies the model of Lee et al., gives the follow equation:

$$
\bar{C} \bar{C}_{g} \nabla^{2} \xi+\bar{k}^{2} \bar{C} \bar{C}_{g} \xi+i \frac{\overline{\partial\left(C C_{g}\right)}}{\partial \omega} \nabla^{2} \frac{\partial \xi}{\partial t}+i \frac{\overline{\partial\left(k^{2} C C_{g}\right)}}{\partial \omega} \frac{\partial \xi}{\partial t}=f_{L}
$$


For a component wave whose angular frequency is $\omega$, we have the following relation:

$$
\frac{\partial \xi}{\partial t}=-i \Delta \omega \xi
$$

where $\Delta \omega=\omega-\bar{\omega}$. The $y$-directional and temporal harmonic terms of $\xi(x, y, t)$ and $f_{L}(x, y, t)$ can be separated as

$$
\begin{aligned}
& \xi(x, y, t)=\tilde{\xi}(x) \exp \left[i\left(k_{y} y-\Delta \omega t\right)\right] \\
& f_{L}(x, y, t)=\tilde{f}_{L}(x) \exp \left[i\left(k_{y} y-\Delta \omega t\right)\right] .
\end{aligned}
$$

Substituting Eqs. (23) and (24) into Eq. (21) and using Eq. (22) give the one-dimensional Helmholtz equation in terms of $\tilde{\xi}$ :

$$
\frac{\partial^{2} \tilde{\xi}}{\partial x^{2}}+k_{x}^{2} \tilde{\xi}=\frac{\tilde{f}_{L}}{\bar{C} \bar{C}_{g}+\Delta \omega \frac{\overline{\partial\left(C C_{g}\right)}}{\partial \omega}}
$$

where the right-hand-side term can be regarded as the source function $\tilde{f}$ in the Helmholtz Eq. (4) and the wave number $k$ is obtained by the dispersion relation of the model (Lee et al., 2004) as 


$$
\frac{k}{\bar{k}}=\sqrt{1+\frac{2\left(\frac{\omega}{\bar{\omega}}-1\right)}{\frac{\bar{C}_{g}}{\bar{C}}+\left(\frac{\omega}{\bar{\omega}}-1\right) \frac{\bar{k}^{2}}{\bar{\omega}} \frac{\partial}{\partial \omega}\left(C C_{g}\right)}}
$$

Following the procedure from Eq. (4) to (8), the surface elevation function $\tilde{\xi}(x)$ corresponding to the source function can be obtained as

$$
\tilde{\xi}(x)=\frac{A D_{L} I}{\bar{C} \bar{C}_{g}+\frac{\overline{\partial\left(C C_{g}\right)}}{\partial \omega} \Delta \omega} \exp \left[i k_{x} \mid x-x_{s}\right]
$$

where $A$ and $I$ are given in Eqs. (7) and (8). On the other hand, the target water surface elevation function $\tilde{\xi}$ is given as

$$
\tilde{\xi}=a \exp \left[i k_{x}\left|x-x_{s}\right|\right]
$$

Thus, the parameter $D_{L}$ in Eq. (27) can be determined by equating $\tilde{\xi}$ in Eqs. (27) and (28) as

$$
D_{L}=\frac{a\left[\bar{C} \bar{C}_{g}+\frac{\overline{\partial\left(C C_{g}\right)}}{\partial \omega} \Delta \omega\right]}{A I}
$$


Thus, the source function $f_{L}$ for Lee et al.'s model is obtained as

$$
f_{L}=\frac{a\left[\bar{C} \bar{C}_{g}+\frac{\overline{\partial\left(C C_{g}\right)}}{\partial \omega} \Delta \omega\right]}{A I} \exp \left[-\beta\left(x-x_{s}\right)^{2}\right] \exp \left[i\left(k_{y} y-\Delta \omega t\right)\right] .
$$

\section{Numerical experiments}

In this section, both monochromatic and random waves are tested for the models of

Suh et al. (1997) and Lee et al. (2003) in horizontally one dimension. And also multidirectional random waves are tested in horizontally two dimensions. For the Suh et al.'s model, the time-derivative terms are discretized by a fourth-order Adams-Moulton predictor-corrector method and the spatial derivative terms are discretized by a threepoint symmetric formula. The Lee et al.'s model equation is discretized by the CrankNicolson method in horizontally one dimension and by the ADI method in horizontally two dimensions.

\subsection{Uni-directional monochromatic waves}


The computational domain consists of an inner domain of $10 L$, where $L$ is the wavelength, and two sponge layers with the thickness of $2.5 \mathrm{~L}$ at the outside boundaries. The center of the source region is located at the center of the inner domain (see Fig. 1). The grid spacing $\Delta x$ is chosen as $\Delta x=L / 20$ and the time step $\Delta t$ is chosen so that the Courant number is $C_{r}=C_{e} \Delta t / \Delta x=0.1$, where $C_{e}$ is the energy velocity (Lee and Suh, 1998), so that a stable solution is guaranteed.

Fig. 1

Firstly, we generate uni-directional monochromatic waves. Fig. 2 and Fig. 3 show numerical solutions of the surface elevation and wave amplitude in shallow water $(k h=0.05 \pi)$ and deep water $(k h=2 \pi)$, respectively, at time of $t=30 T$, where $T$ is the wave period. In the figures, the solutions are normalized with the target wave amplitude $a_{0}$. Both wave amplitudes and wavelengths are almost equal to the target values. Also, wave energy is dissipated at the sponge layer almost perfectly.

Fig. 2 
Secondly, we test whether the waves pass through the wave generation band without any numerical disturbances. We remove the sponge layer at the right boundary, and reduce the computational domain to $8 L$. The relative water depth is $k h=2 \pi$, and Lee et al.'s model is used. Fig. 4 shows a snapshot of water surface elevations at $t=30 T, 30 T+T / 4,30 T+2 T / 4,30 T+3 T / 4$. The waves reflected from the right boundary do not make any numerical disturbances when passing through the wave generation region around $x / L=0$, while wave energy is well absorbed in the left sponge layer.

Fig. 4

\subsection{Uni-directional random waves}

In generating random waves and predicting the propagation of the waves, the frequency of carrier waves must be chosen so as to give minimal errors in a wide range of local wave frequencies. As the difference between the component wave frequency and the carrier one becomes larger, the model yields larger errors in both wave phase and energy. The accuracy of the dispersion relation of the model determines the accuracy of the model. 
Lee et al. (2004) found that the dispersion relation of the Suh et al.'s model is accurate at higher frequencies, while the dispersion relation of the Lee et al.'s model does not have a particular range of high accuracy. Therefore, for the Suh et al.'s model, the peak frequency is chosen as the carrier frequency which is relatively lower in whole frequency range. For the Lee et al.'s model, the weight-averaged frequency is chosen as the carrier frequency which is higher than the peak frequency.

The TMA shallow-water spectrum is used as the target spectrum (Bouws et al., 1985) :

$$
S(f)=\alpha g^{2}(2 \pi)^{-4} f^{-5} \exp \left[-1.25\left(f / f_{p}\right)^{-4}\right] \gamma^{\exp \left[-\left(f / f_{p}-1\right)^{2} / 2 \sigma^{2}\right]} \phi_{k}(f, h)
$$

where $\alpha$ is a spectral parameter, $\gamma$ is the peak enhancement factor, $\sigma$ is the spectral width parameter, and the Kitaigordskii shape function, $\phi_{k}(f, h)$ incorporates the effect of finite water depth. $\alpha=7.57 \times 10^{-4}, \gamma=2$, and the peak frequency of input wave, $f_{p}=$ $0.767 \mathrm{~Hz}$ is used.

The computational domain has an inner domain of $10 L_{\max }\left(L_{\max }\right.$ is the maximum wavelength resulting from the cutoff incident frequency spectrum), and the center of 
wave generation band is located at a distance of $L_{\max }$ from the up-wave boundary of the inner domain (see Fig. 5). Two sponge layers with the thickness of $S=2.5 L_{\max }$ are placed at the outside boundaries.

Fig. 5

To ensure the resolution in space and time as well as the numerical stability, the frequency range of incident waves is confined to 0.6 to $1.4 \mathrm{~Hz}$. The grid size is chosen as $\Delta x=L_{\min } / 30.3$ ( $L_{\min }$ is the minimum wavelength resulting from the cutoff incident frequency spectrum) and the time step is chosen for the maximum Courant number $C_{r \max }=C_{e \max } \Delta t / \Delta x$ to be 0.1 .

Uni-directional random waves are generated by superposing all the source functions corresponding to all the component waves:

$$
\begin{aligned}
& f_{S}(x, y, t)=\sum_{m}-\frac{a_{m} \bar{C} \bar{C}_{g}}{\omega_{m} A_{m} I_{m}} \exp \left[-\beta\left(x-x_{s}\right)^{2}\right] \exp \left[i\left(k_{y_{m}} y-\omega_{m} t+\varepsilon_{m}\right)\right] \\
& f_{L}(x, y, t)=\sum_{m} \frac{a_{m}\left[\bar{C} \bar{C}_{g}+\frac{\overline{\partial\left(C C_{g}\right)}}{\partial \omega} \Delta \omega_{m}\right]}{A_{m} I_{m}} \exp \left[-\beta\left(x-x_{s}\right)^{2}\right] \exp \left[i\left(k_{y_{m}} y-\Delta \omega_{m} t+\varepsilon_{m}\right)\right]
\end{aligned}
$$


where $m$ denotes the number of frequency components, $a_{m}$ is the amplitude of a component wave, $\varepsilon_{m}$ is the phase angle which is randomly distributed between 0 and $2 \pi$, and $A_{m}$ and $I_{m}$ are given in Eqs. (7) and (8).

The water surface elevations are recorded at a distance of $L_{\max }$ up-wave from the down-wave boundary of the inner domain. The surface elevations are recorded from $t=100 T_{p}$ at every $0.05 \mathrm{~s}$, where $T_{p}$ is the peak wave period, and the total number of samples is 16384. Figs. 6 and 7 compare simulated wave spectra with the target in shallow water $(k h=0.05 \pi)$ and deep water $(k h=2 \pi)$, respectively. The agreement between the target and computed spectrum is excellent in whole frequency ranges.

Fig. 6

Fig. 7

\subsection{Multi-directional random waves}

Finally, we carry out the test of multi-directional random waves. Lee and Suh (1998) tested the line source method to generate multi-directional random waves. In this study, we use the same wave conditions as they used. The TMA shallow-water spectrum is used 
as a target spectrum, and the conditions of input waves are $T_{p}=10 \mathrm{~s}, h=35 \mathrm{~m}$, $\alpha=0.003523, \gamma=20$. To insure the resolution in space and time and also the numerical stability, the frequency range of incident waves is confined between $0.086 \mathrm{~Hz}$ and $0.123 \mathrm{~Hz}$ that covers $85 \%$ of the total energy and gives the significant wave height of $H_{s}=4.24 \mathrm{~m}$.

The amplitude of each wave component is given by $a_{i, j}=\sqrt{2 S\left(f_{i}\right) G\left(f_{i}, \theta_{j}\right) \Delta f_{i} \Delta \theta_{j}}$ where the directional spreading function $G(f, \theta)$ is obtained by the Fourier series representation for the wrapped normal spreading function as:

$G(f, \theta)=\frac{1}{2 \pi}+\frac{1}{\pi} \sum_{n=1}^{N} \exp \left[-\frac{\left(n \sigma_{m}\right)^{2}}{2}\right] \cos \left[n\left(\theta-\theta_{m}\right)\right]$

where $N(=20)$ is the number of terms in the series, the mean wave direction $\theta_{m}$ is $0^{\circ}$, and the directional spreading parameter $\sigma_{m}$ is $10^{\circ}$. These conditions of input waves are the same as the M1 case in Lee and Suh (1998). The representative directions are $-14^{\circ}$, $-5^{\circ}, 0^{\circ}, 5^{\circ}, 14^{\circ}$, and these directional components are taken to have equal area of directional spreading function. The amplitude of each wave component is $a_{i, j}=\sqrt{2 S\left(f_{i}\right) \Delta f_{i} / 5} \quad(j=1, \cdots, 5)$. 
We generate multi-directional random waves by superposing all the source functions corresponding to all the component waves as:

$f_{S}(x, y, t)=\sum_{m} \sum_{n}-\frac{a_{m, n} \bar{C} \bar{C}_{g}}{\omega_{m} A_{m, n} I_{m, n}} \exp \left[-\beta\left(x-x_{s}\right)^{2}\right] \exp \left[i\left(k_{y_{m, n}} y-\omega_{m} t+\varepsilon_{m, n}\right)\right]$

$f_{L}(x, y, t)=\sum_{m} \sum_{n} \frac{a_{m, n}\left[\bar{C} \bar{C}_{g}+\frac{\overline{\partial\left(C C_{g}\right)}}{\partial \omega} \Delta \omega_{m}\right]}{A_{m, n} I_{m, n}} \exp \left[-\beta\left(x-x_{s}\right)^{2}\right] \exp \left[i\left(k_{y_{m, n}} y-\Delta \omega_{m} t+\varepsilon_{m, n}\right)\right]$

where $m$ and $n$ denote the components of different frequency and direction, respectively, $\varepsilon_{m, n}$ is the phase angle which is randomly distributed between 0 and $2 \pi$, and $A_{m, n}$ and $I_{m, n}$ are given in Eqs. (7) and (8).

The computational domain consists of an inner domain of $3.5 L_{\max } \times 3 L_{\max }$ and the sponge layers with the thickness $S=2.5 L_{\max }$ at four outside boundaries. The centerline of the source band is located at a distance of $0.5 L_{\max }$ from the up-wave boundary of the inner domain. Fig. 8 shows a schematic diagram of the computational domain. The water surface elevation $(\eta)$ and its slopes in the $x$ - and $y$-directions $(\partial \eta / \partial x, \partial \eta / \partial y)$ are recorded at a point of $\left((x, y)=\left(L_{\max }, 1.5 L_{\max }\right)\right)$ of the inner domain from $10 T_{p}$ at every 0.32 second until the total number of samples becomes 8192 . 
Fig. 8

In order to calculate the directional wave spectrum, we analyzed the data by using the maximum entropy principle method (MEP). Fig. 9 shows the target spectrum and the two-dimensional wave spectra calculated from the recorded data. And, Fig. 10 shows the frequency spectra and the directional spreading function for the peak frequency. All the results show that the agreement between the target spectrum and computed one is good, and the source function method is very efficient even for generation of multi-directional random waves.

Fig. 9

Fig. 10

\section{Conclusions}

Following the method of Wei et al. (1999), we have developed a wave generation method using a source function for the time-dependent extended mild-slope equations of Suh et al. (1997) and Lee et al. (2003). Numerical simulations of uni-directional 
monochromatic waves showed that the waves were properly generated for an arbitrary water depth. The wave reflection test showed that the waves passing through the source region did not cause any numerical disturbances.

In simulating random waves, the peak frequency was chosen as a carrier frequency for the Suh et al.'s model, and the weight-averaged frequency for the Lee et al.'s model. For uni-directional random waves, the computed wave spectrum was nearly identical to the target one for an arbitrary relative water depth, and multi-directional random waves were also generated as desired. The source functions obtained in this study for the extended mild-slope equations of Suh et al. and Lee et al. can also be used for the mild-slope equations of Radder and Dingemans (1985) and Kubo et al. (1992), respectively, because the source functions were derived based on the assumption of a constant water depth. In conclusion, we verified that the source function of the Helmholtz equation may be used in generating waves in any refraction-diffraction equation models.

\section{Appendix A. The method of Green's function}

In order to obtain the relationship between the source function $f(x, y, t)$ and the wave 
property $F(x, y, t)$, a particular solution of the differential equation including a source function should be obtained. The differential equation after excluding the component of waves propagating in the $y$-direction is given by

$\frac{\partial^{2} \tilde{F}}{\partial x^{2}}+k_{x}^{2} \tilde{F}=\tilde{f}(x)$

where the relations between $F$ and $\tilde{F}$ and those between $f$ and $\tilde{f}$ are given in Eqs. (2) and (3), respectively. Homogeneous solutions of Eq. (4) corresponding to progressive waves are given by

$\tilde{F}_{h}=\exp \left( \pm i k_{x} x\right)$

In order to obtain a particular solution, we seek a Green's function $G(\xi, x)$ which satisfies

$\frac{\partial^{2} G}{\partial \xi^{2}}+k_{x}^{2} G=\delta(\xi-x)$

where $\xi$ and $x$ are regarded as the active and fixed variables, respectively, and $\delta$ is the Dirac delta function. The wave property and the Green's function should satisfy the 
radiation boundary conditions given by

$\begin{array}{llll}\frac{\partial^{n} G}{\partial \xi^{n}}=\left(+i k_{x}\right)^{n} G, & \frac{\partial^{n} \tilde{F}}{\partial \xi^{n}}=\left(+i k_{x}\right)^{n} \tilde{F} & \text { as } & \xi \rightarrow+\infty \\ \frac{\partial^{n} G}{\partial \xi^{n}}=\left(-i k_{x}\right)^{n} G, & \frac{\partial^{n} \tilde{F}}{\partial \xi^{n}}=\left(-i k_{x}\right)^{n} \tilde{F} & \text { as } & \xi \rightarrow-\infty\end{array}$

Integrating Eq. (38) with respect to $\xi$ from $x-0$ to $x+0$, we have

$\left.\frac{\partial G}{\partial \xi}\right|_{\xi=x-0} ^{\xi=x+0}+k_{x}^{2} \int_{x-0}^{x+0} G d \xi=1$

If the Green's function is continuous at $\xi=x$, Eq. (40) becomes

$$
\left.\frac{\partial G}{\partial \xi}\right|_{\xi=x-0} ^{\xi=x+0}=1
$$

The Green's function that satisfies the radiation conditions (39), and the continuity condition (41) at $\xi=x$ is given by

$$
G(\xi, x)= \begin{cases}G_{+}=-\frac{i}{2 k_{x}} \exp \left[i k_{x}(\xi-x)\right], & \xi>x \\ G_{-}=-\frac{i}{2 k_{x}} \exp \left[i k_{x}(x-\xi)\right], & \xi<x\end{cases}
$$


where $A=-i / 2 k_{x}$. Multiplying Eq. (38) by $\tilde{F}(\xi)$ and integrating with respect to $\xi$ from $-\infty$ to $+\infty$ give

$$
\int_{-\infty}^{+\infty} \tilde{F}(\xi)\left[\frac{\partial^{2} G(\xi, x)}{\partial \xi^{2}}+k_{x}^{2} G(\xi, x)\right] d \xi=\int_{-\infty}^{+\infty} \tilde{F}(\xi) \delta(\xi-x) d \xi
$$

Integrating by parts and using the boundary conditions and the definition of the delta function give

$$
\begin{aligned}
\tilde{F}(x) & =\int_{-\infty}^{+\infty} G(\xi, x) \tilde{f}(\xi) d \xi \\
& =\int_{-\infty}^{x} G_{-}(\xi, x) \tilde{f}(\xi) d \xi+\int_{x}^{+\infty} G_{+}(\xi, x) \tilde{f}(\xi) d \xi
\end{aligned}
$$

The arbitrary function $\tilde{f}$ can be selected as a smooth Gaussian shape given by

$$
\tilde{f}(x)=D \exp \left[-\beta\left(x-x_{s}\right)^{2}\right]
$$

where the parameter $D$ is the source function amplitude, and $\beta$ is related to the width of the source function. The source function is negligibly small except in the vicinity of $x_{s}$. Therefore, in an interested region $\left(x>x_{s}\right)$, the second integral in the righ-hand side of 
Eq. (44) is negligible, and thus we have

$$
\begin{aligned}
\tilde{F}(x) & \cong \int_{-\infty}^{x} G_{-}(\xi, x) \tilde{f}(\xi) d \xi \\
& \cong \int_{-\infty}^{\infty} G_{-}(\xi, x) \tilde{f}(\xi) d \xi \\
& =A D I \exp \left[i k_{x}\left(x-x_{s}\right)\right]
\end{aligned}
$$

where $I$ is defined by

$$
I=\int_{-\infty}^{+\infty} \exp \left(-\beta \xi^{2}\right) \exp \left(-i k_{x} \xi\right) d \xi=\sqrt{\frac{\pi}{\beta}} \exp \left(-\frac{k_{x}^{2}}{4 \beta}\right)
$$

For waves that are generated at a point $x=x_{s}$ and propagate in the opposite region $\left(x<x_{s}\right)$, a similar approach is used to give

$$
\tilde{F}(x) \cong \int_{x}^{+\infty} G_{+}(\xi, x) \tilde{f}(\xi) d \xi \cong A D I \exp \left[i k_{x}\left(x_{s}-x\right)\right]
$$




\section{References}

Berkhoff, J.C.W., 1972. Computation of combined refraction-diffraction. Proc. 13th Int.

Conf. On Coastal Eng. Conf., ASCE, Vancouver, Canada, pp. 471-490.

Bouws, E., Günther, H., Rosenthal, W., Vincent, C.L., 1985. Similarity of the wind wave spectrum in finite depth 1. Spectral form. Journal of Geophysical Research 90(C1), 975-986.

Chamberlain, P.G., Porter, D., 1995. The modified mild-slope equation. Journal of Fluid Mechanics 291, 393-407.

Copeland, G.J.M., 1985. A practical alternative to the mild-slope wave equation. Coastal Engineering 9, 125-149.

Kubo, Y., Kotake, Y., Isobe, M., Watanabe, A., 1992. Time-dependent mild slope equation for random waves. Proc. 23rd Int. Conf. On Coastal Eng., ASCE, Venice, Italy, pp. 419-431.

Larsen, J., Dancy, H., 1983. Open boundaries in short wave simulations - a new approach. Coastal Engineering 7, 285-297.

Lee, C., Cho, Y.-S., Yum, K., 2001. Internal generation of waves for extended Boussinesq equations. Coastal Engineering 42, 155-162.

Lee, C., Kim, G., Suh, K.D., 2003. Extended mild-slope equation for random waves. 
Coastal Engineering 48, 277-287.

Lee, C., Kim, G., Suh K.D., 2004. Comparison of time-dependent extended mild-slope equations for random waves. Proc. Asian and Pacific Coasts 2003.

Lee, C., Suh, K.D., 1998. Internal generation of waves for time-dependent mild-slope equations. Coastal Engineering 34, 35-57.

Madsen, P. A. Larsen, J., 1987. An efficient finite-difference approach to the mild-slope equation, Coastal Engineering 11, 329-351.

Massel, S.R., 1993. Extended refraction-diffraction equation for surface waves. Coastal Engineering 19, 97-126.

Nishimura, H., Maruyama, K., Hirakuchi, H., 1983. Wave field analysis by finite difference method. Proc. 30th Japanese Conf. Coastal Eng., pp. 123-127 (in Japanese).

Nwogu, O., 1993. Alternative form of Boussinesq equations for nearshore wave propagation. Journal of Waterway, Port, Coastal and Ocean Engineering 119, 618638.

Peregrine, D.H., 1967. Long waves on a beach. Journal of Fluid Mechanics 27, 815-827.

Radder, A.C., Dingemans, M.W., 1985. Canonical equations for almost periodic, weakly nonlinear gravity waves. Wave Motion 7, 473-485.

Smith, R., Sprinks, T., 1975. Scattering of surface waves by a conical island. Journal of 
Fluid Mechanics 72, 373-384.

Suh, K.D., Lee, C., Park, W.S., 1997. Time-dependent equations for wave propagation on rapidly varying topography. Coastal Engineering 32, 91-117.

Wei, G., Kirby, J.T., Sinha, A., 1999. Generation of waves in Boussinesq models using a source function method. Coastal. Engineering 36, 271-299. 


\section{Captions of figures}

Fig. 1. Computational domain for generating uni-directional monochromatic waves.

Fig. 2. Normalized water surface elevations and amplitudes of uni-directional monochromatic waves at $t=30 T$ in shallow water with $k h=0.05 \pi$; solid line $=$ water surface elevation, dashed line = wave amplitude. (a) Suh et al.'s model, (b) Lee et al.'s model.

Fig. 3. Normalized water surface elevations and amplitudes of uni-directional monochromatic waves at $t=30 T$ in deep water with $k h=2 \pi$; solid line $=$ water surface elevation, dashed line = wave amplitude. (a) Suh et al.'s model, (b) Lee et al.'s model.

Fig. 4. Snapshots of water surface elevations at $\mathrm{t}=30 \mathrm{~T}, 30 \mathrm{~T}+\mathrm{T} / 4,30 \mathrm{~T}+2 \mathrm{~T} / 4$, $30 \mathrm{~T}+3 \mathrm{~T} / 4$.

Fig. 5. Computational domain for generating uni-directional random waves.

Fig. 6. Frequency spectra of uni-directional random waves with $k_{p} h=0.05 \pi$; solid line = numerical solution, dashed line $=$ target. (a) Suh et al.'s model, (b) Lee et al.'s model.

Fig. 7. Frequency spectra of uni-directional random waves with $k_{p} h=2 \pi$; solid line $=$ numerical solution, dashed line = target. (a) Suh et al.'s model, (b) Lee et al.'s model.

Fig. 8. Computational domain for generating multi-directional random waves. 
Fig. 9. Computed 2D directional wave spectra. (a) Target spectrum, (b) Suh et al.'s model, (c) Lee et al.'s model.

Fig. 10. (a) Frequency spectra and (b) directional spreading functions (solid line $=$ target spectrum, dashed line $=$ Suh et al.'s model, dash-dotted line $=$ Lee et al.'s model). 


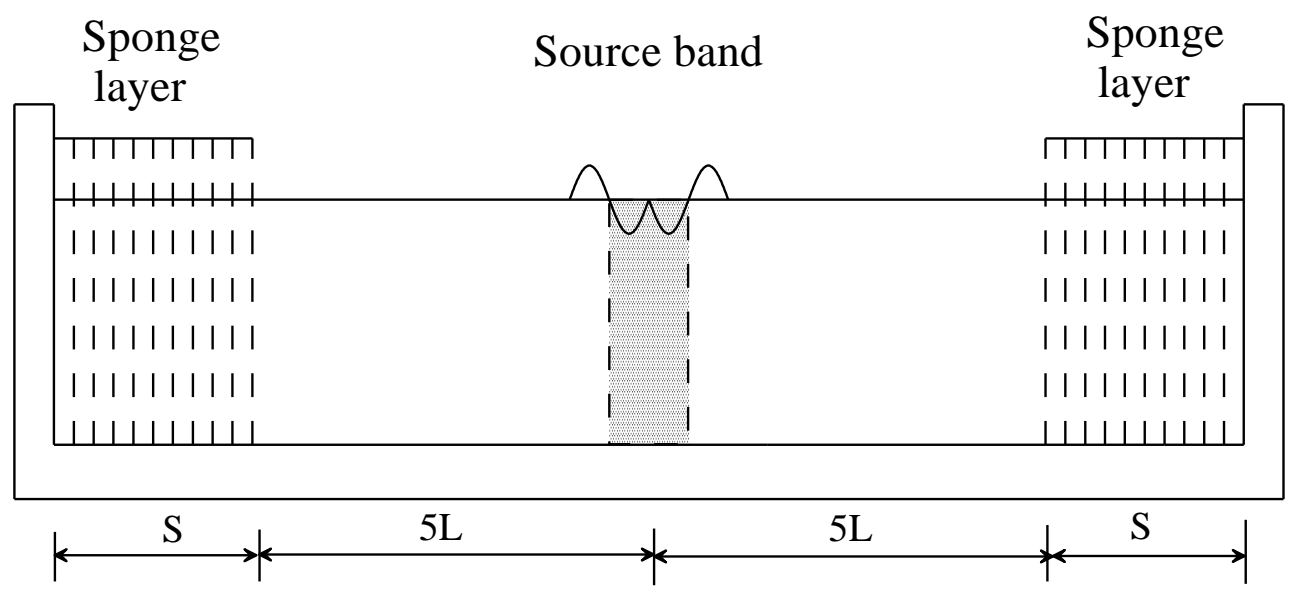

Fig. 1. Computational domain for generating uni-directional monochromatic waves. 
(a)

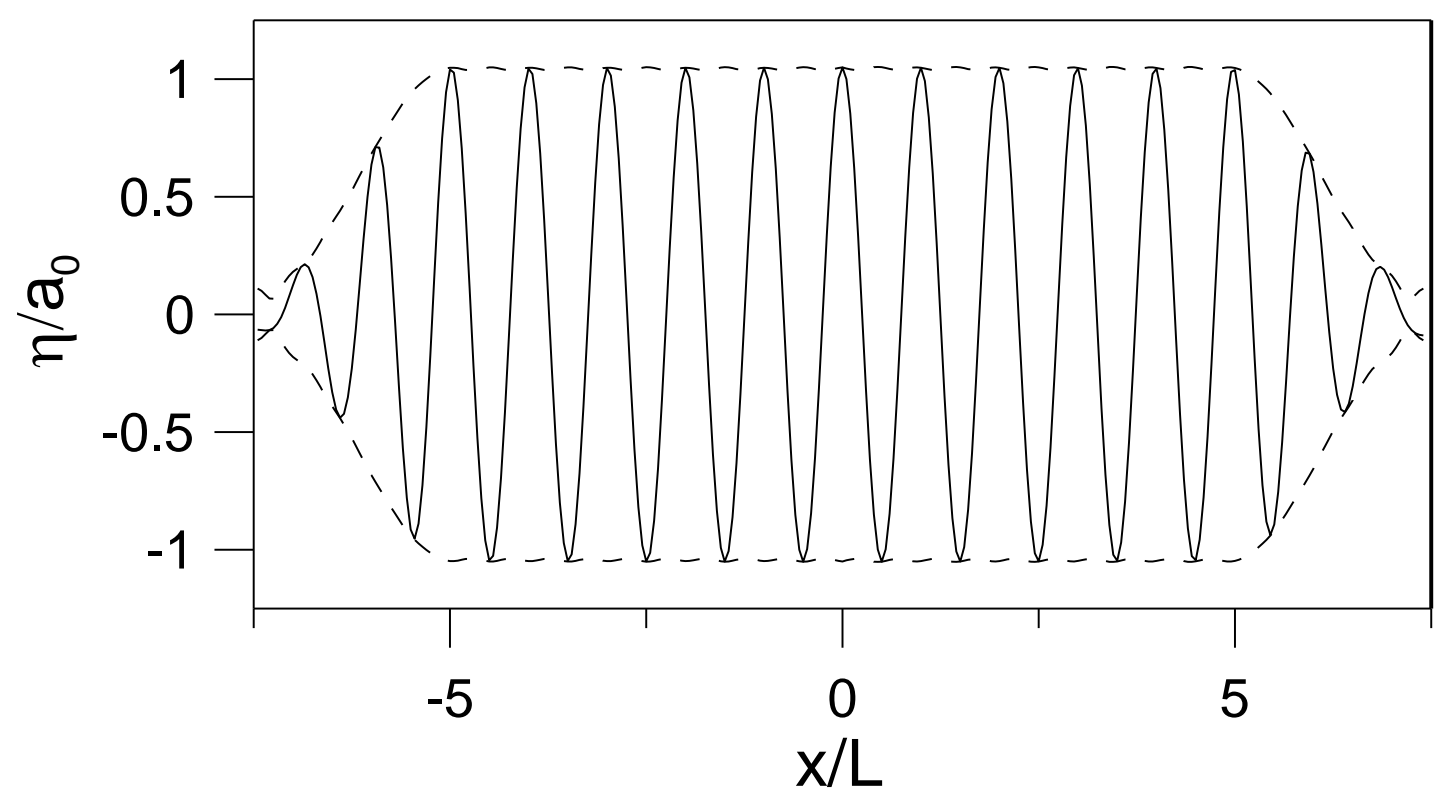

(b)

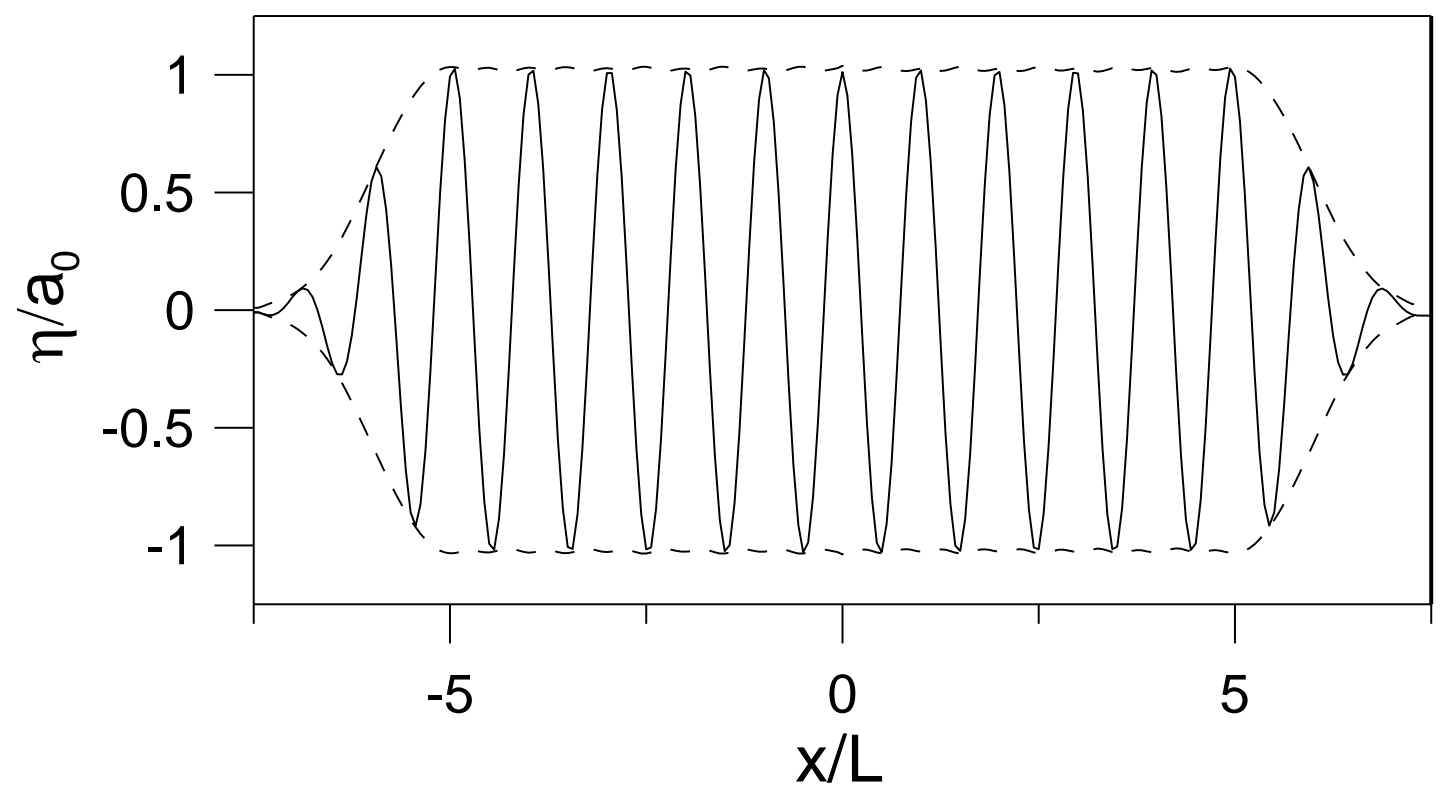

Fig. 2. Normalized water surface elevations and amplitudes of uni-directional monochromatic waves at $t=30 T$ in shallow water with $k h=0.05 \pi$; solid line $=$ water surface elevation, dashed line = wave amplitude. (a) Suh et al.'s model, (b) Lee et al.'s model. 
(a)

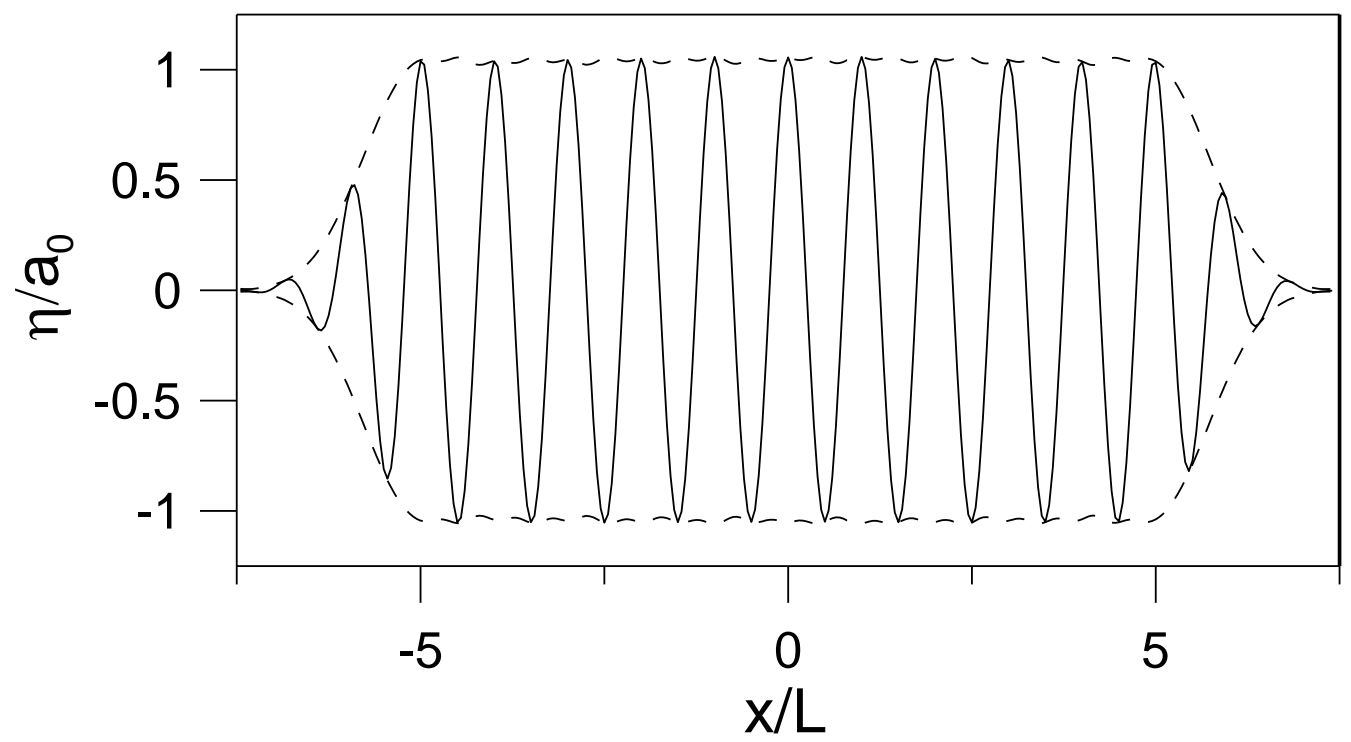

(b)

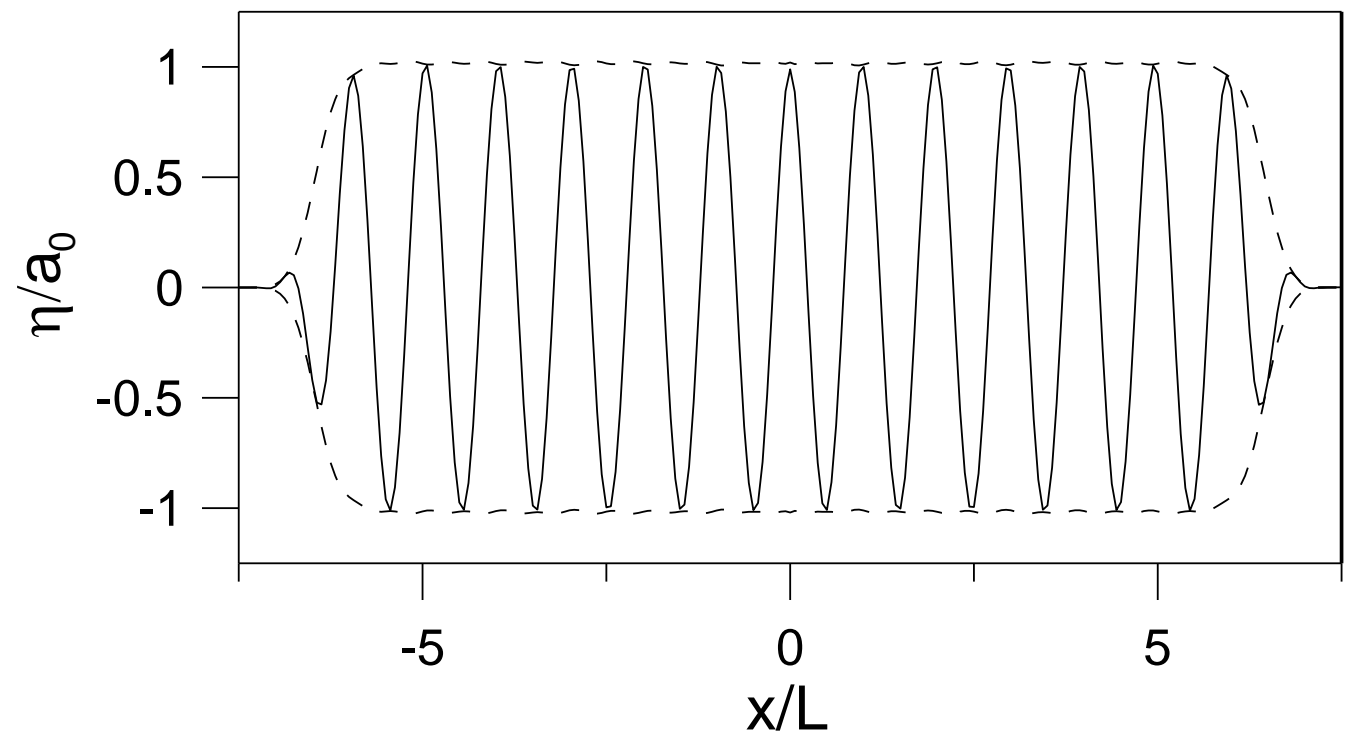

Fig. 3. Normalized water surface elevations and amplitudes of uni-directional monochromatic waves at $t=30 T$ in deep water with $k h=2 \pi$; solid line $=$ water surface elevation, dashed line = wave amplitude. (a) Suh et al.'s model, (b) Lee et al.'s model. 


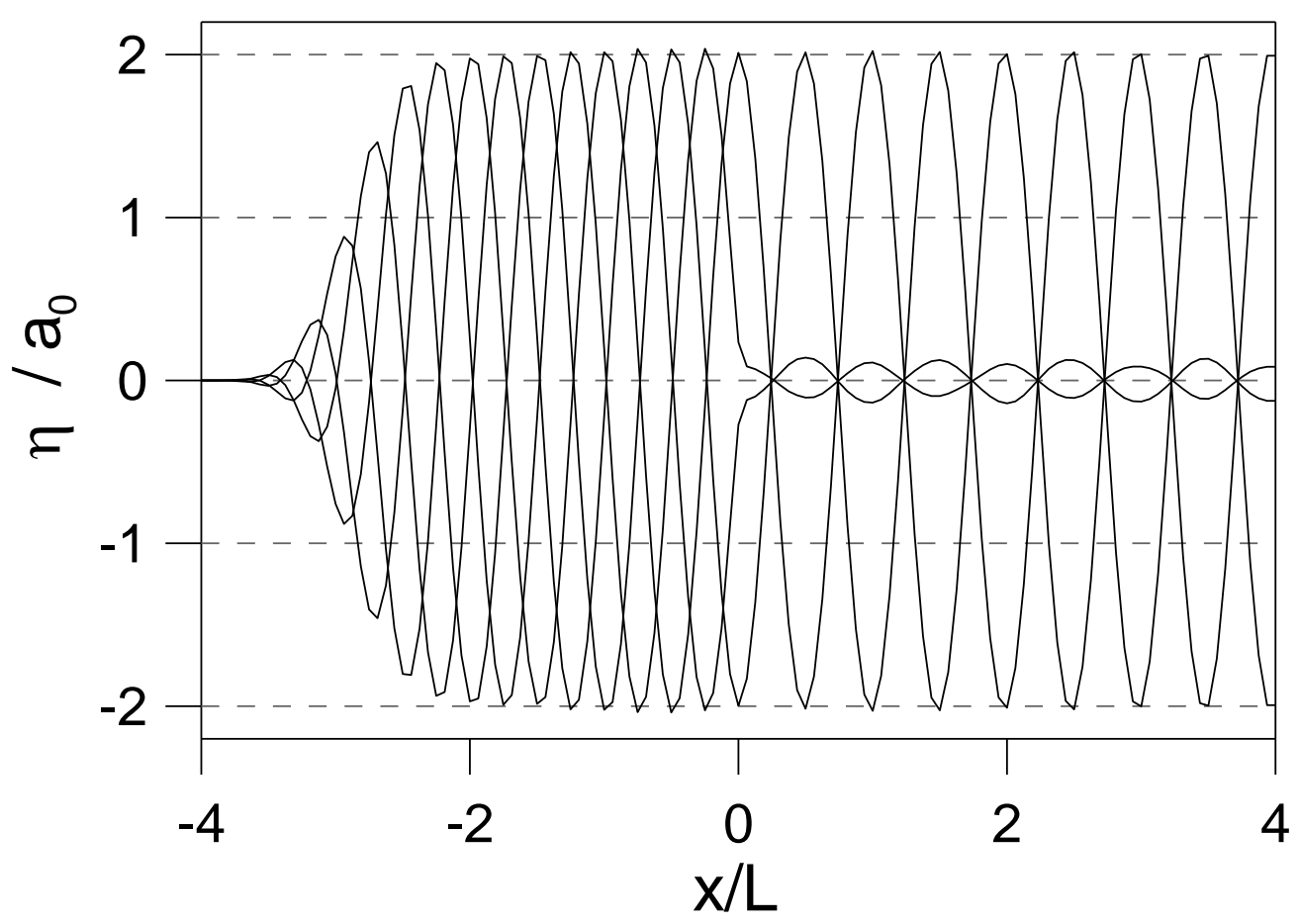

Fig. 4. Snapshots of water surface elevations at $\mathrm{t}=30 \mathrm{~T}, 30 \mathrm{~T}+\mathrm{T} / 4,30 \mathrm{~T}+\mathrm{T} / 2$, $30 \mathrm{~T}+3 \mathrm{~T} / 4$. 


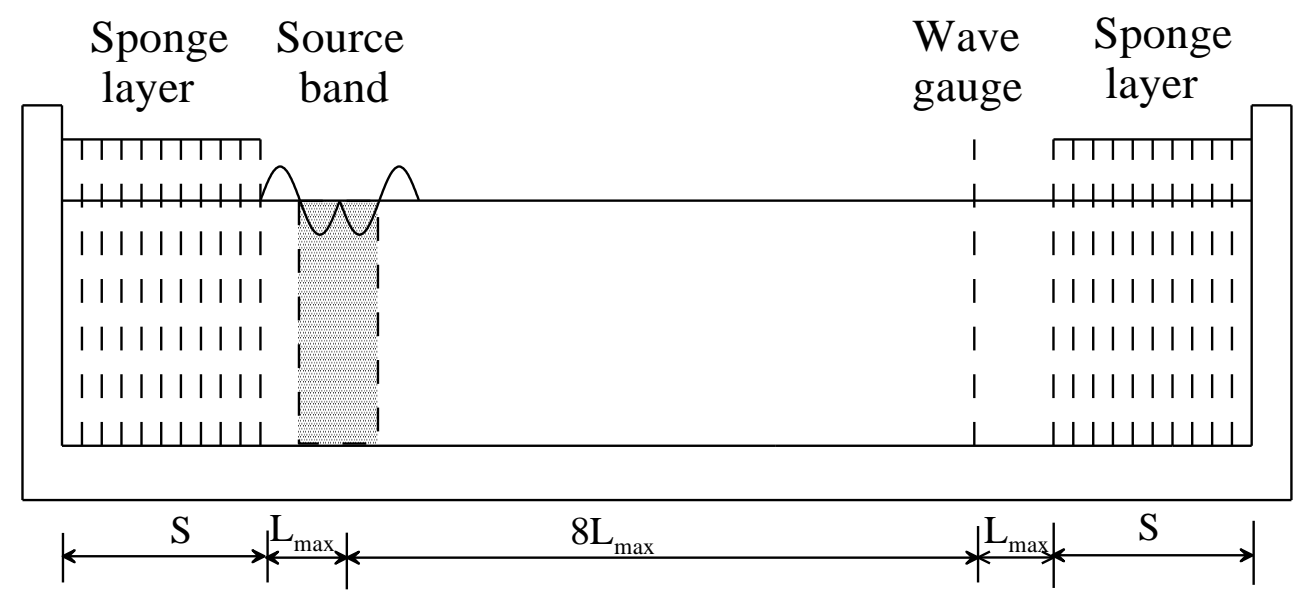

Fig. 5. Computational domain for generating uni-directional random waves. 
(a)

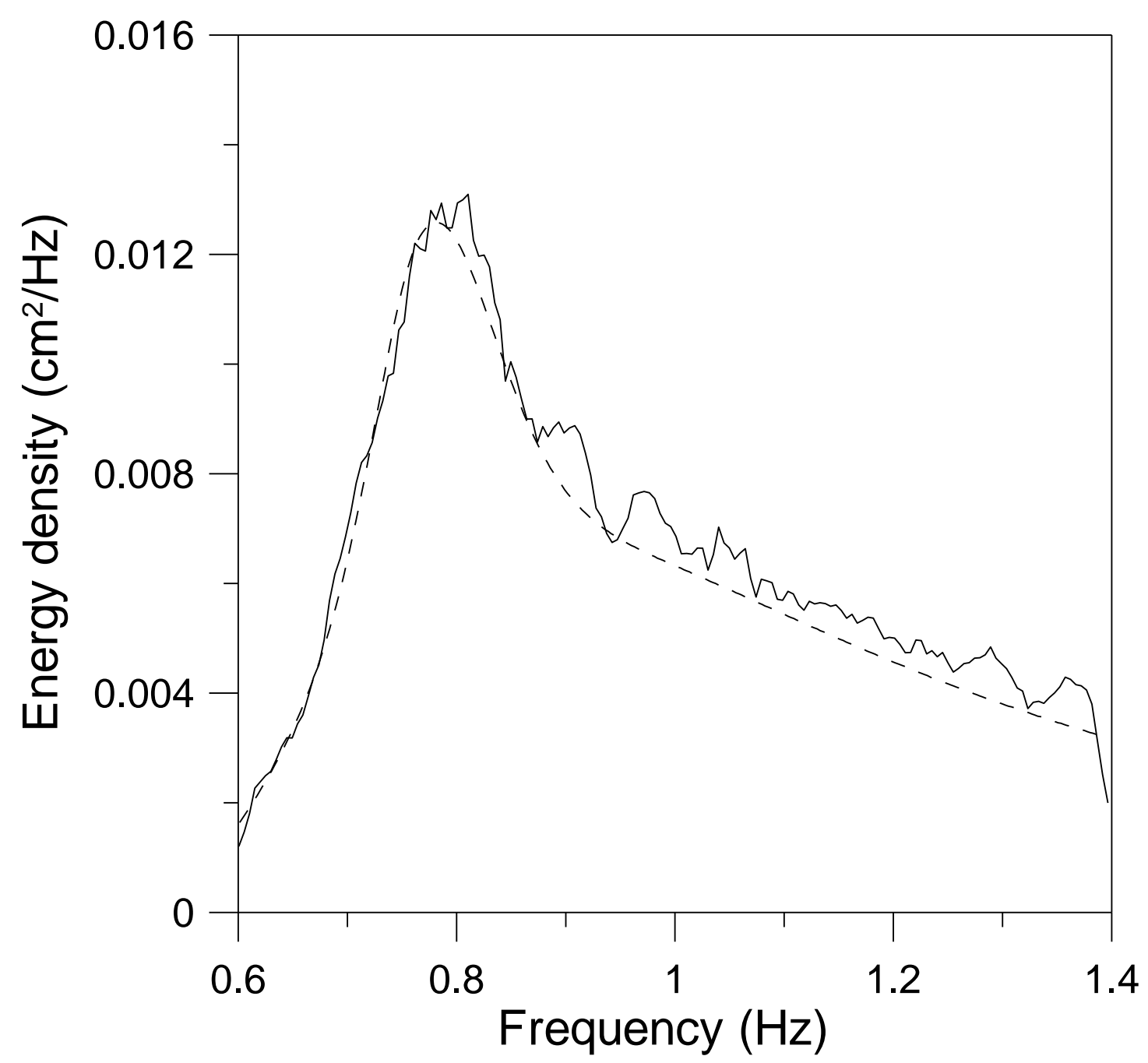

Fig. 6. Frequency spectra of uni-directional random waves with $k_{p} h=0.05 \pi$; solid line $=$ numerical solution, dashed line $=$ target. (a) Suh et al.'s model, (b) Lee et al.'s model. 
(b)

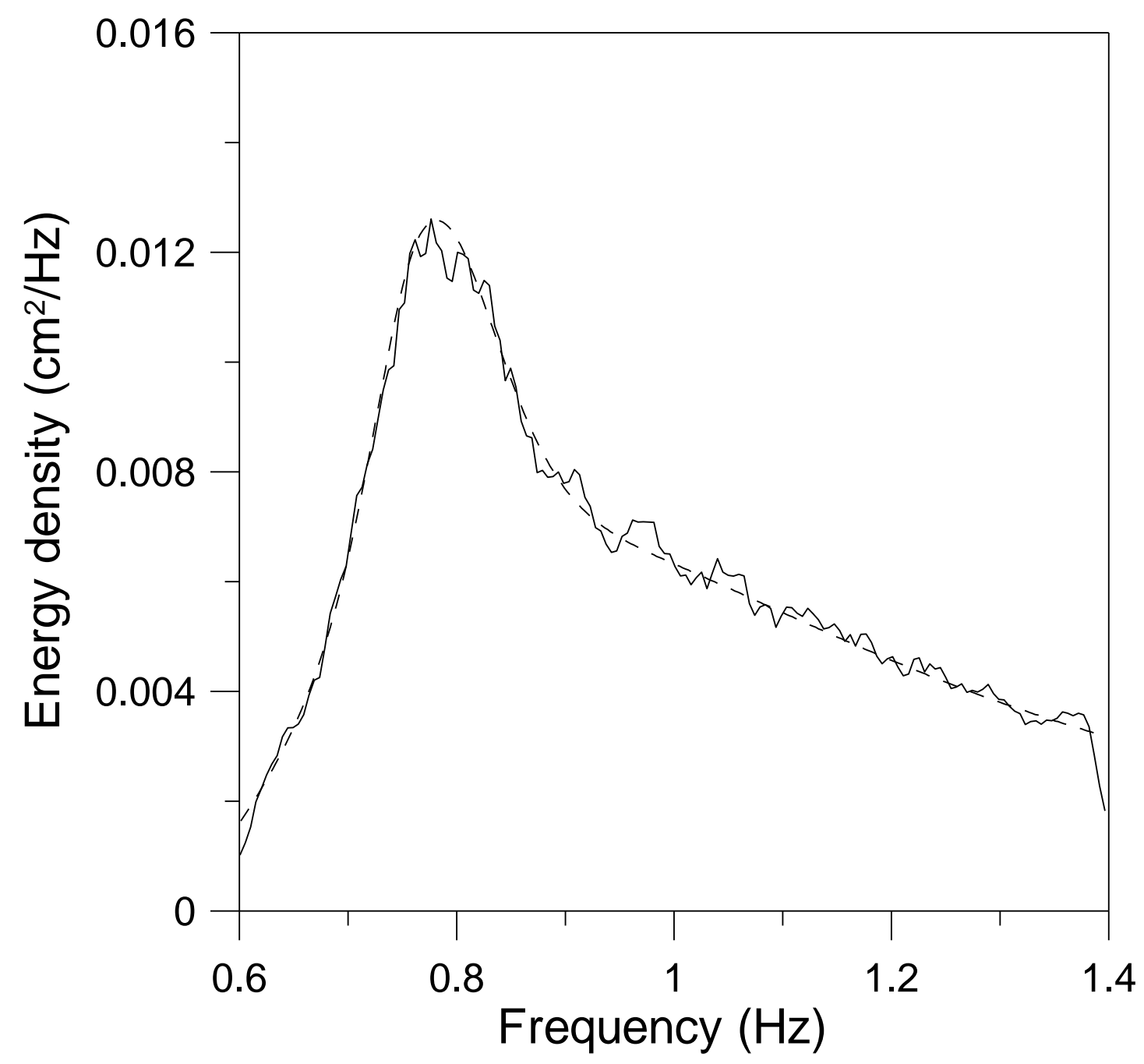

Fig. 6. (Continued). 
(a)

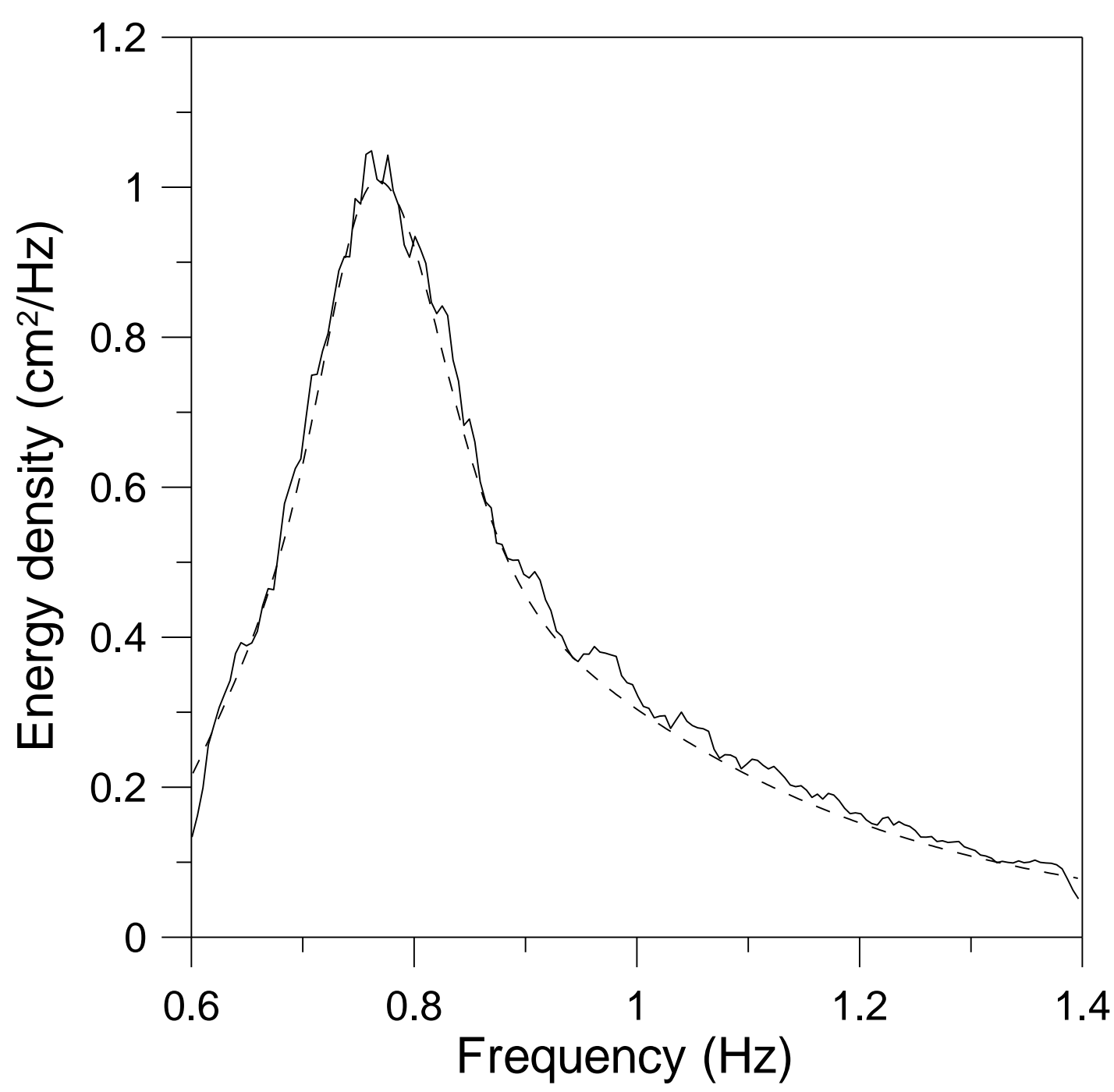

Fig. 7. Frequency spectra of uni-directional random waves with $k_{p} h=2 \pi$; solid line $=$ numerical solution, dashed line = target. (a) Suh et al.'s model, (b) Lee et al.'s model. 
(b)

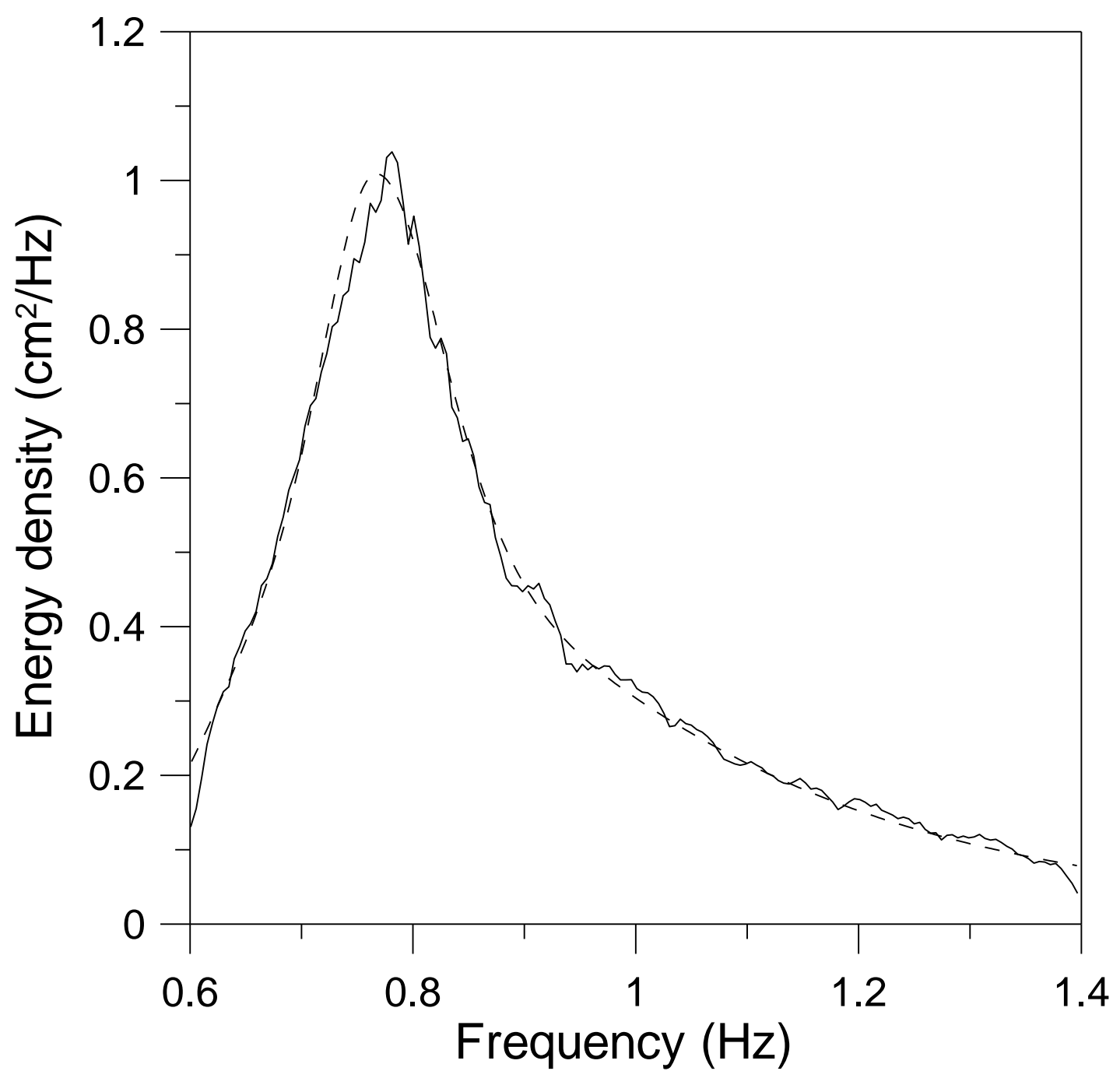

Fig. 7. (Continued). 


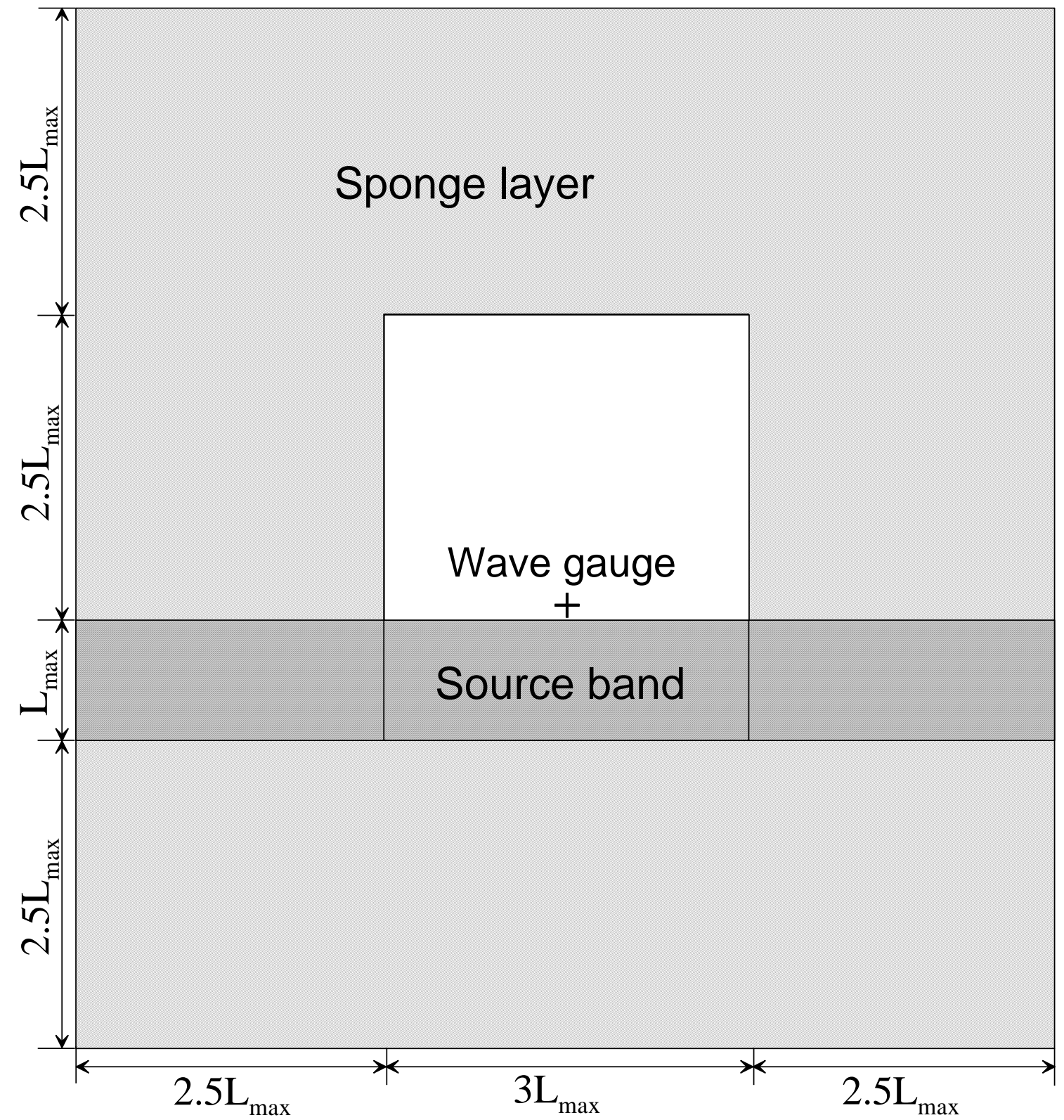

Fig. 8 Computational domain for generating multi-directional random waves. 
(a)

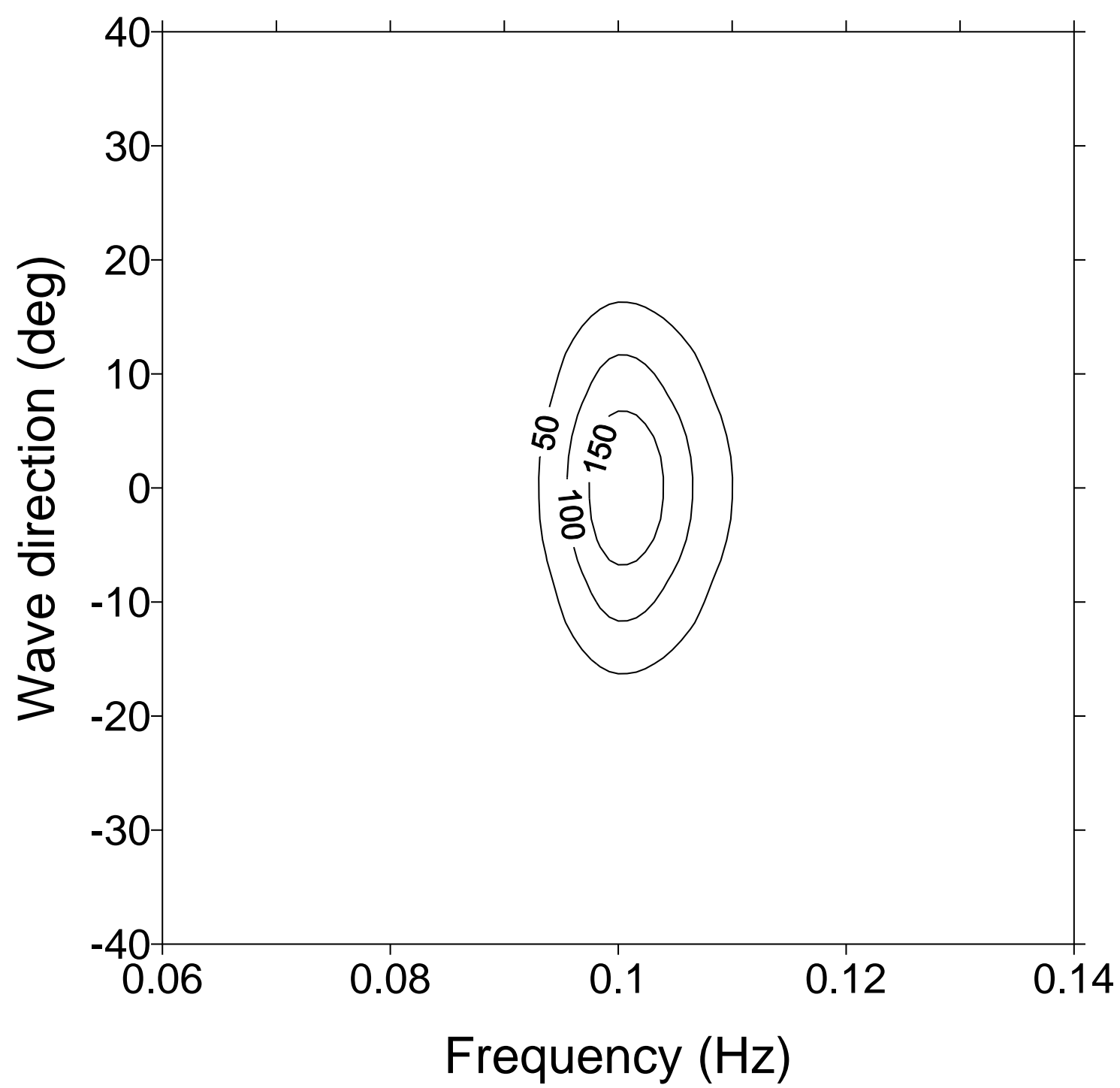

Fig. 9. Computed 2D directional wave spectra. (a) Target spectrum, (b) Suh et al.'s model, (c) Lee et al.'s model. 
(b)

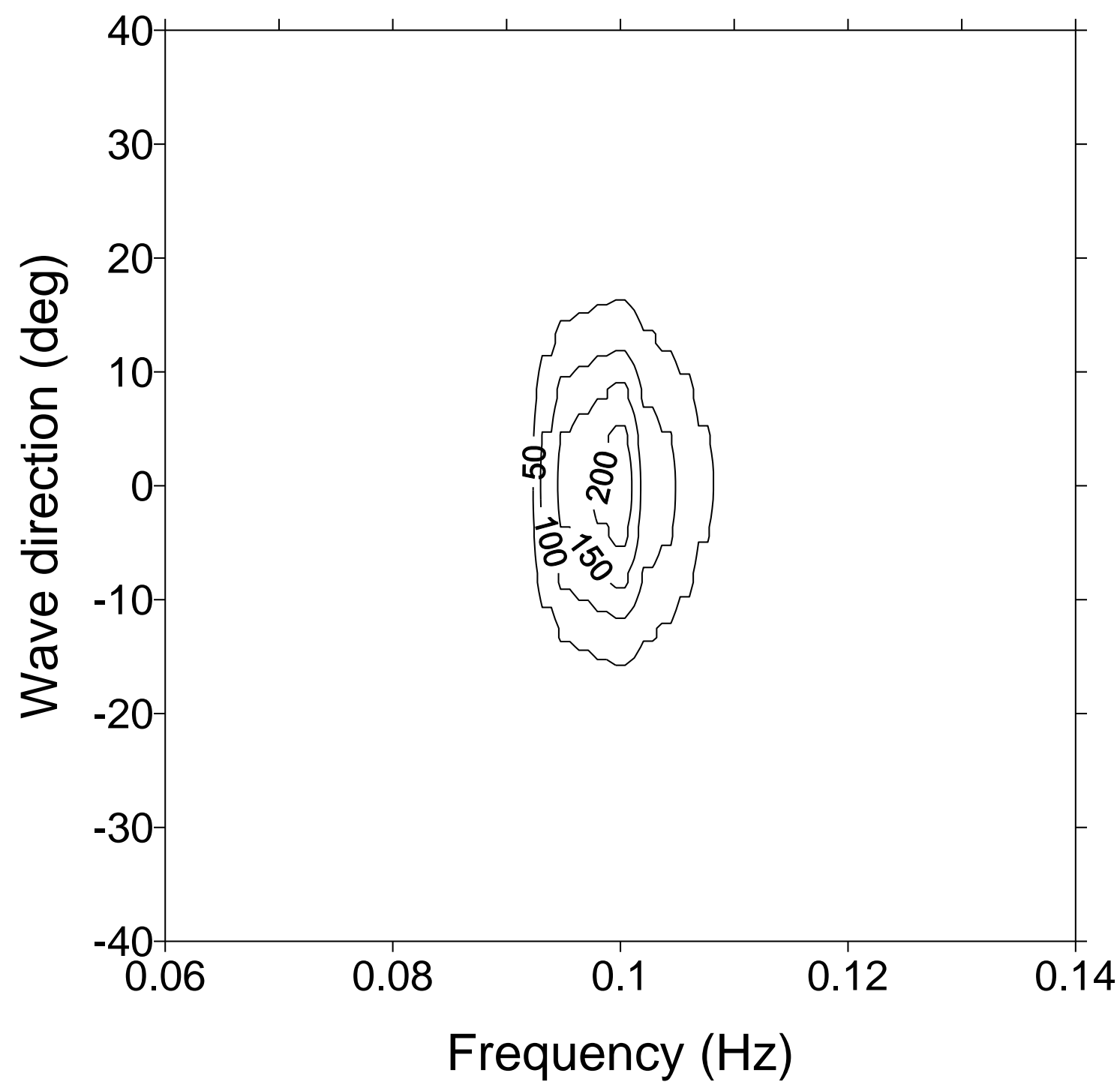

Fig. 9. (Continued). 
(c)

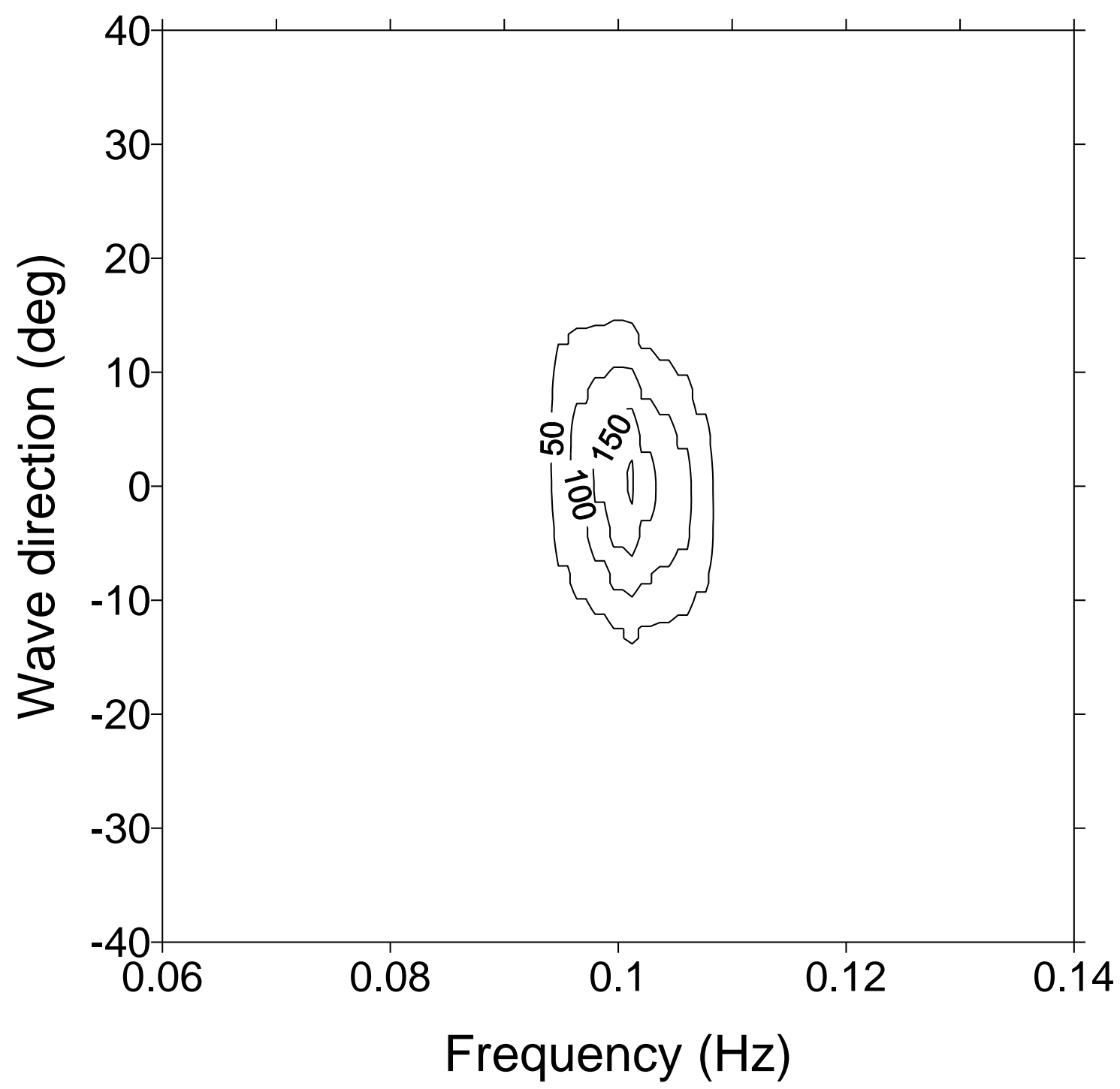

Fig. 9. (Continued). 
(a)

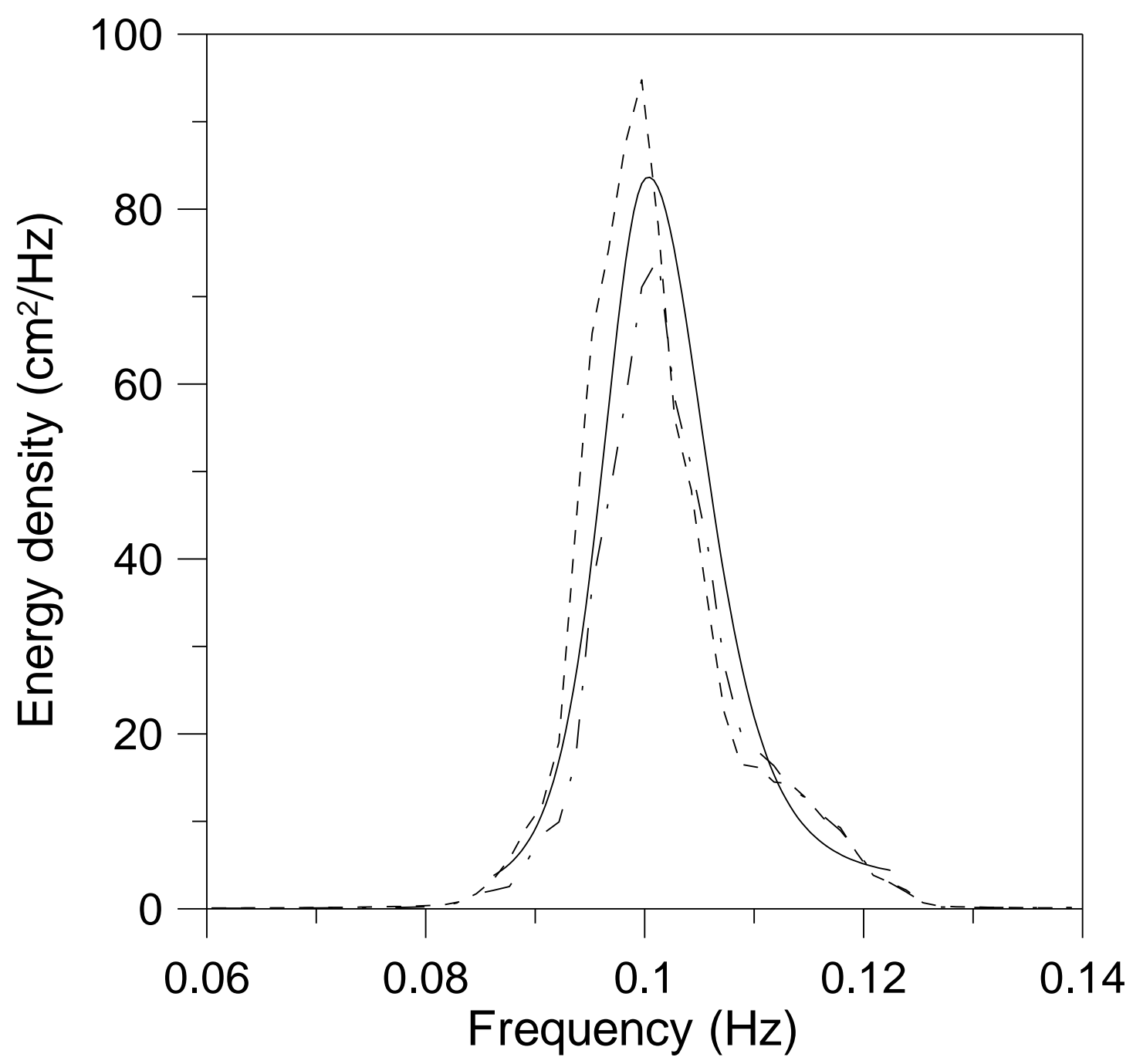

Fig. 10. (a) Frequency spectra and (b) directional spreading functions (solid line $=$ target spectrum, dashed line $=$ Suh et al.'s model, dash-dotted line= Lee et al.'s model) . 
(b)

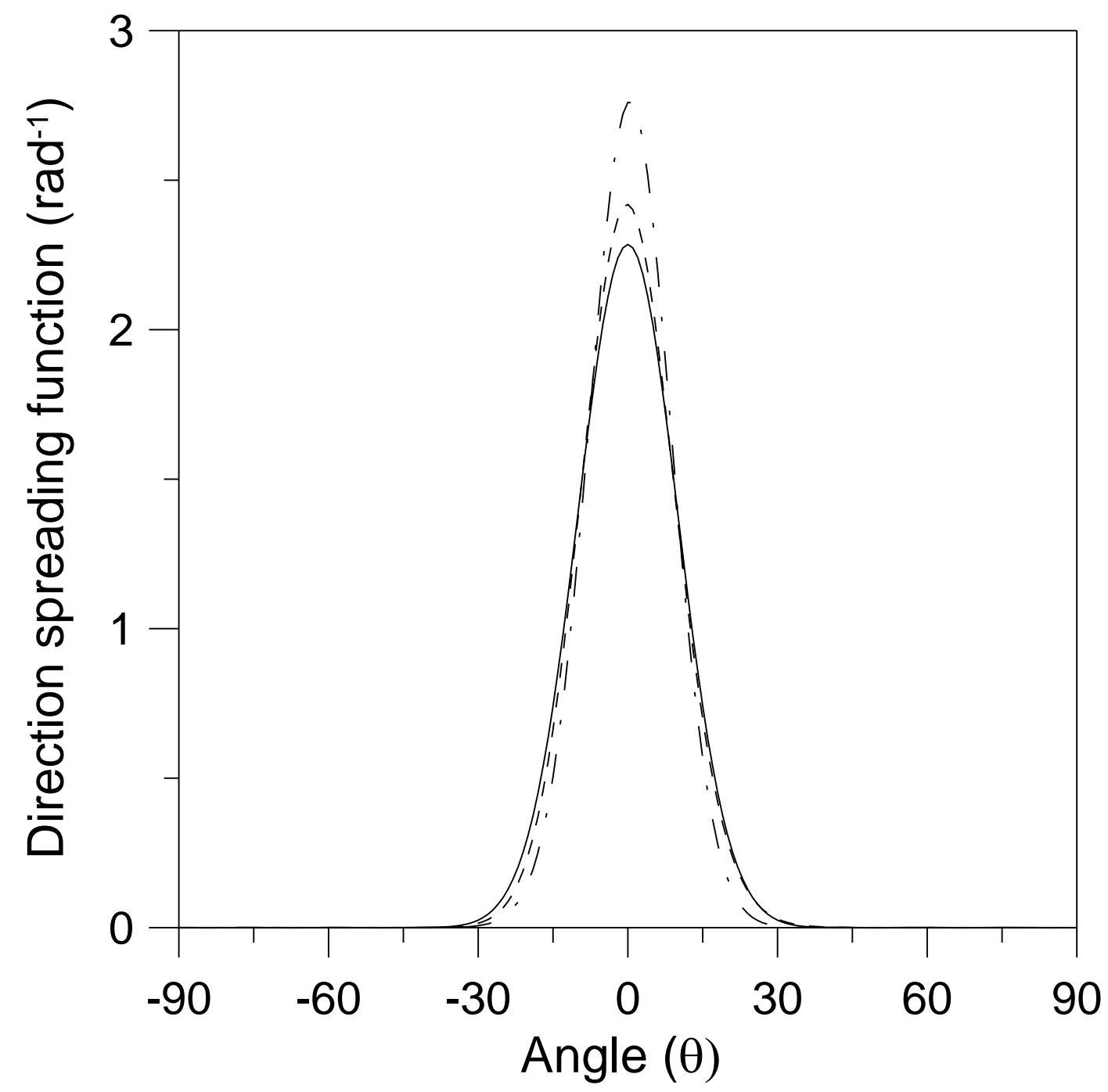

Fig. 10. (Continued). 